\title{
EFICIÊNCIA RELATIVA DE FERTILIZANTES FOSFATADOS NO CRESCIMENTO INICIAL DE EUCALIPTO CULTIVADO EM SOLOS DO CERRADO(1)
}

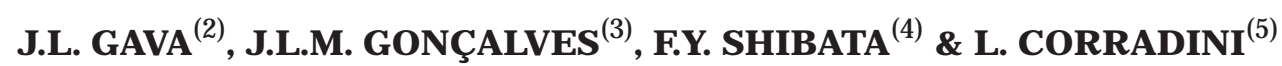

\begin{abstract}
RESUMO
Tendo como objetivo avaliar a eficiência relativa do superfosfato si mples (SS), fosfato parcialmente acidulado (FAPS) e termofosfato magnesiano (TE RM) como fonte de $\mathbf{P}$ e outros nutrientes para o cultivo de plantações de eucalipto, em dois solos da região dos Cerrados, uma areia quartzosa $\left(50 \mathrm{~g} \mathrm{~kg}^{-1}\right.$ de argila) álica e um latossolo vermel ho-escuro ( $230 \mathrm{~g} \mathrm{~kg}^{-1}$ de argila) álico, foi realizado este experimento, sob condições de casa de vegetação, na ESALQ/USP, Piracicaba (SP), em 1993. Subamostras do $A Q$ receberam 0, 75, 150 e $300 \mathrm{mg} \mathrm{kg}^{-1}$ de $P$ e, do LE, 0, 125, 250 e $500 \mathrm{mg} \mathrm{kg}^{-1}$ de $P$ no solo. As doses de $P$ foram estimadas com base na solubilidade dos adubos; SS e FAPS, em citrato de amônio, e TE RM, em ácido cítrico $20 \mathrm{~g} \mathrm{L-1.} \mathrm{E} \mathrm{m}$ cada vaso (5 $\mathrm{kg}$ de solo), deixou-se uma planta de Eucalyptus grandis por 90 dias após a emergência e desbaste das plântulas. Paralelamente ao experimento em casa de vegetação, subamostras de todos os tratamentos, com $2 \mathbf{~ k g}$ de solo, foram incubadas no interior de sacos plásticos por $0,10,20,40,60$ e 80 dias. Ao térmi no de cada período de incubação, os solos eram amostrados e analisados. Mediante 0 cálculo de índices de eficiência relativa entre os adubos, verificou-se que o TE RM foi superior $64 \%$ ao SS e $57 \%$ ao FAPS no solo AQ. No LE, o SS foi superior $55 \%$ ao TER M e 34\%ao FAPS. O TE RM foi a fonte de P que promoveu os maiores acréscimos do nível de fertilidade dos solos, com expressiva elevação do pH do solo, redução das concentrações de $\mathrm{Al}$ e aumento daquelas de $\mathrm{Ca}$ e $\mathrm{Mg}$. Os ganhos de produção das mudas de eucalipto foram altamente correlacionados com a absorção de $P, K$, $\mathrm{Ca}, \mathrm{Mg}$ e Zn. O TE RM proporcionou as maiores absorções desses nutrientes no solo AQ e, as menores, de P, Ca e Zn no LE. As distintas respostas de crescimento para os adubos avaliados, em ambos os solos, sobretudo para TERM e SS, foram atribuídas, em grande parte, aos efeitos secundários das fontes de $P$ sobre a fertilidade dos solos.
\end{abstract}

Termos de indexação: eucalipto, eficiência de adubos fosfatados, fosfato parcialmente acidulado, superfosfato si mples, termofosfato magnesiano e região dos Cerrados.

\footnotetext{
(1) Parte do trabal ho de pesquisa do primeiro e do terceiro autores, como bolsistas de iniciação científica da FAPESP, no Departamento de Ciências Florestais da ESALQ. Recebido para publicação em junho de 1996 e aprovado em junho de 1997.

(2) Engenheiro Florestal da Cia Suzano, Caixa Postal 228, CEP 18200-000 I tatinga (SP).

(3) Professor Associado do Departamento de Ciências Florestais da ESALQ, Caixa Postal 9, CEP 13418-900 Piracicaba (SP). Bolsista do CNPq. E-mail: jlmgonca@carpa.ciagri.usp.br.

(4) Estudante de Engenharia Florestal da ESALQ, Caixa Postal 9, CEP 13418-900 Piracicaba (SP).

(5) Engenheiro Florestal da Consultoria Florestal, Rua Vicente de Carvalho, 364, sala 110, CEP 14020-040 Ribeirão Preto (SP).
} 


\title{
SUMMARY: RELATIVE EFFICIENCY OF PHOSPHATE FERTILIZERS ON THE INITIAL GROWTH OF EUCALYPT IN SOILS FROM CERRADO REGION
}

\begin{abstract}
The objective of this study was to assess the relative efficiency of single superphosphate (SS), partially acidulated rock phosphate (PARP) and fused magnesium thermal phosphate (MTP) as sources of $P$ and other nutrients for eucalypt plantations in two soils of the cerrado region: an alic quartz-sand ( $50 \mathrm{~g} \mathrm{~kg}^{-1}$ of clay) and an al ic dark-red latosol (230 g kg-1 of clay). Experiments were carried out under greenhouse conditions at the University of São Paulo, Piracicaba, State of São Paulo, Brazil, in 1993. Sub-samples of quartz-sand received 0, 75, 150 and $300 \mathrm{mg} \mathrm{kg}^{-1}$ of $P$ in the soil, and dark-red latosol, $0,125,250$ and $500 \mathrm{mg} \mathrm{kg}^{-1}$ of $P$ in the soil. The P doses wereestimated on the basis of thesolubility of thefertilizers SS and PARP in ammonium citrate, and MTP in $20 \mathrm{~g} \mathrm{~L}-1$ citric acid. Eucalyptus grandis seedlings weregrown, individually, in pots containing $5 \mathrm{~kg}$ of soil, for 90 days after emergence Paralle to thegrenhouse experiment, soil sub-samples of each treatment, $2 \mathrm{~kg}$ weight, wereincubated in plastic bags for periods of $0,10,20,40,60$ and 80 days. At the end of each incubation period, they were sampled and analysed. The relative efficiency indexes between the fertilizers indicated that MTP was superior $64 \%$ to SS and $57 \%$ to PARP, in the quartz-sand. In thedark-red latosol, SS was superior $55 \%$ to MTP and $34 \%$ to PARP. The MTP was the P source that induced the higher increase in the soil fertility level, with a significant $\mathrm{pH}$ increase, reduction of $\mathrm{Al}$ concentration and increase in $\mathrm{Ca}$ and $\mathrm{Mg}$ concentration. The increasing yield of the eucalypt seedlings was highly correlated to $\mathrm{P}, \mathrm{K}, \mathrm{Ca}, \mathrm{Mg}$ and $\mathrm{Zn}$ uptake MTP provided the highest uptake of these nutrients in quartz-sand and the lowest uptake of $\mathrm{P}, \mathrm{Ca}$ and $\mathrm{Zn}$ in dark-red latosol. The distinct growing responses to the fertilizers, particularly to MTP and to SS, in both soils, were largely dueto secondary effects of theP sources on the soil fertility.
\end{abstract}

Index terms: eucalypt, relative efficiency of P fertilizers, single superphophate (SS), partially acidulate rock phosphate (PARP), magnesium thermal phosphate (MTP), cerrados.

\section{INTRODUÇÃO}

Os florestamentos com eucalipto e Pinus têm expandido consideravelmente no Brasil, sobretudo em regiões onde os solos apresentam baixa fertilidade, como nos Cerrados. Estima-se que a área atual florestada seja superior a 6,5 milhões de hectares, sendo mais de $75 \%$ ocupadas com poucas espécies de eucalipto. A tendência de crescimento da área florestada é grande, em virtude da expansão das indústrias de celul ose e papel, chapas e agl omerados, serraria, siderurgia, cerâmica e outras. A mai oria das áreas em cultivo e grande parte daquelas que serão agregadas respondem positivamente à adubação, principal mente a fosfatada.

Entre as fontes de $P$, as solúveis têm sido as mais promissoras, promovendo acréscimos economicamente viáveis de produtividade (Herbert, 1983, Herbert \& Schönau, 1989, Barros et al., 1990, Gonçalves, 1995). Com relação às fontes pouco solúveis, indicadas, preferencialmente, para culturas perenes e pastagens (Ballard, 1984, Goedert \& Souza, 1984), as pesquisas têm mostrado que as respostas aos fosfatos naturais, como única fonte de $P$, às vezes são pouco ou não compensadoras para florestamentos com eucalipto (Rezende et al., 1982, Leal et al., 1988). Geralmente, pela magnitude das respostas, particularmente quando as áreas de plantio são distantes das indústrias, as fontes solúveis podem ser mais viáveis. $\mathrm{O}$ uso de fosfatos naturais tem si do promissor quando associado com fontes solúveis de P. Em virtude da alta demanda inicial do eucalipto por $\mathrm{P}$ (N ovais et al., 1982), deve-se realizar a adubação fosfatada com a aplicação localizada de uma fonte solúvel por ocasião do plantio, antecedida pela adição da fonte natural aplicada a lanço (Barros et al., 1990). A despeito dos poucos trabalhos de pesquisa realizados sobre otema, sobretudo quando se considera a grande diversidade de condições ambientais onde se encontram os florestamentos, a importância da "adubação de arranque" para Eucalyptus grandis foi confirmada pelas pesquisas de Rezende et al. (1982), Dantas (1988) e Leal et al. (1988). A rocha fosfatada tende a apresentar maior eficiência como fonte de $\mathrm{P}$ em solos de textura argilosa, acidez el evada, alta capacidade de fixação de fósforo e baixos níveis de P e Ca.

Graças aos baixos preços da madeira (bitola fina) de eucalipto e aos al tos custos dos adubos, muito representativos no custo final de implantação e manejo das florestas, as dosagens de adubos, economicamente viáveis, são muito baixas quando comparadas à mai oria das culturas agrícolas, mesmo considerando a grande capacidade de adaptação do eucal i pto em sol os distróficos. Nessas circunstâncias, devem-se escolher as fontes de $\mathrm{P}$ com as menores relações (ud de $\mathrm{P}_{2} \mathrm{O}_{5}$ solúvel/ud monetária de custo), mas que, ao mesmo tempo, disponibilizem às árvores outros nutrientes essenciais, carentes nos solos do Cerrado, e demandados em grande quantidade como, por exemplo, Ca eS.

O benefício de um adubo, portanto, não se deve basear apenas na disponibilidade de seu nutriente 
primário, mas nos seus efeitos gerais sobrefertilidade do sol o e nutrição das árvores. Nesse sentido, a mel hor fonte não é, necessariamente, aquela que forneça as maiores quantidades de $P$ nutricionalmente ativos, mas a que forneça, também, outros nutrientes secundários limitantes no solo, ou que propicie às árvores relações adequadas entre nutrientes essenciais no solo, com efeito sinér gico de um sobre o outro durante a absorção. Segundo Gonçal ves (1995), a reposição da maior parte do Ca removido com a madeira em plantações deP inus seria reposta somente com o uso de fontes de $\mathrm{P}$ ricas em $\mathrm{Ca}$.

Desse contexto, o presente trabalho teve como objetivo avaliar a eficiência relativa de três fertilizantes fosfatados como fonte de $\mathrm{P}$ e outros nutrientes secundários para o cultivo do eucali pto, em dois solos da região dos Cerrados.

\section{MATERIAL E MÉTODOS}

Para a instalação do experimento, foram usadas amostras da camada de $0-20 \mathrm{~cm}$ de profundidade de dois solos sob vegetação de cerrado, no município de Luiz Antônio (SP). Um deles é uma areia quartzosa (AQ) álica e, o outro, um latossolo vermelho-escuro (LE ) álico, textura média. A pós a col eta das amostras, os solos foram secos à sombra, peneirados (malha de $2 \mathrm{~mm}$ ), homogeneizados e caracterizados física e quimicamente. Suas características granulométricas são as seguintes: $A Q$, areia $=930$, silte $=20$ e argila $=50 \mathrm{~g} \mathrm{~kg}^{-1}$ e, do LE, areia $=730$, silte $=40 \mathrm{e}$ argila $=230 \mathrm{~g} \mathrm{~kg}^{-1}$. Suas características químicas eram: $A Q$, matéria orgânica $=19 \mathrm{~g} \mathrm{~kg}^{-1} ; \mathrm{pH}$ em $\mathrm{CaCl}_{2}$ $0,01 \mathrm{~mol} \mathrm{~L}^{-1}(1: 2,5)=3,7$; P-resina $=16 \mathrm{mg} \mathrm{dm}^{-3}$; $\mathrm{K}=0,2 ; \mathrm{Ca}=1,3 ; \mathrm{Mg}=0,3 ; \mathrm{H}+\mathrm{Al}=34,0 \mathrm{mmol}_{\mathrm{c}} \mathrm{dm}^{-3} \mathrm{e}$ $\mathrm{V}=5 \%$ e, para o LE , matéria orgânica $=25 \mathrm{~g} \mathrm{~kg}^{-1}$; $\mathrm{pH}$ em $\mathrm{CaCl}_{2}$ 0,01 mol L-1 $(1: 2,5)=3,8$; P-resina $=$ $7 \mathrm{mg} \mathrm{dm}-3 ; \mathrm{K}=0,3 ; \mathrm{Ca}=1,2 ; \mathrm{Mg}=0,4 ; \mathrm{H}+\mathrm{Al}=$ $72,0 \mathrm{mmol}_{\mathrm{c}} \mathrm{dm}^{-3} \mathrm{eV}=2 \%$, avaliadas segundo método descrito por Raij et al. (1987).

\section{Cultivo de mudas de Eucalyptus grandis}

Subamostras dos dois solos receberam diferentes dosagens de fósforo, de forma a conseguir níveis crescentes de fósforo disponível nos solos. O AQ recebeu adições de $P$ correspondentes a 0, 75, 150 e $300 \mathrm{mg} \mathrm{kg}^{-1}$ de $P$ no solo e o LE , 0, 125, 250 e $500 \mathrm{mg} \mathrm{kg}^{-1}$ de $\mathrm{P}$ no solo. Como fontes de fósforo, usaram-se três adubos comerciais: superfosfato simples (forma granulada), fosfato natural parcialmente solubilizado (FAPS), obtido a partir do concentrado fosfático de Araxá tratado com $350 \mathrm{~kg}$ de ácido sulfúrico por tonelada de concentrado, e termofosfato magnesiano em pó, Yoorin, (Quadro 1). As doses de P aplicadas ao sol o foram estimadas a partir do teor de $P$ solúvel dos adubos: em CNA + água, para oSS eFAPS, eem ácido cítrico a $20 \mathrm{~g} \mathrm{~L}-1$, para o TERM. Os adubos foram homogeneizados com as amostras de solo. Além das adições de $P$, os sol os receberam uma adubação básica correspondente a $100 \mathrm{mg} \mathrm{kg}^{-1}$ de $\mathrm{N}$ no solo e $100 \mathrm{mg} \mathrm{kg}^{-1}$ de $\mathrm{K}$ no solo, tendo como fontes o sulfato de amônio e o cloreto de potássio.

As subamostras de sol o, após receber as adubações mencionadas, foram acondicionadas em vasos plásticos com capacidade de $5 \mathrm{~kg}$ de solo, os quais foram irrigados com água destilada, procurando-se manter o teor de água do solo próximo à capacidade de campo. Deixou-se crescer, em cada vaso, uma planta de Eucalyptus grandis.

Como adubação de cobertura, aplicou-se, em todos os tratamentos, 45 dias após o plantio da semente, uma solução contendo micronutrientes (4 mg de Zn; $1 \mathrm{mg}$ de $\mathrm{B}$; 1,5 mg de Fe; $1 \mathrm{mg}$ de $\mathrm{Mn}$; $1 \mathrm{mg}$ de $\mathrm{Cu}$ e 0,1 mg de $\mathrm{Mo} \mathrm{kg}^{-1}$ de solo). A partir de então, em interval os de dez dias até o corte das mudas, todos os vasos receberam $25 \mathrm{mg} \mathrm{kg}^{-1}$ de N, em forma de sol ução preparada com sulfato de amônio p.a.

Os tratamentos, em quatro repetições, foram dispostos num fatorial $3 \times 4 \times 2$ (três fontes defósforo, quatro doses de $\mathrm{P}$ e dois solos), no delineamento de blocos casualizados, desenvolvendo-se o experimento em casa de vegetação. As plantas foram cultivadas por 90 dias após a emergência.

O material vegetal da parte aérea das plantas foi seco à temperatura de $65^{\circ} \mathrm{C}$ até peso constante. Determinado seu peso, o material foi moído e mineralizado por via úmida, empregando-se a digestão nitroperclórica, segundo método descrito por Sarruge \& Haag (1974). As determinações das concentrações de macro e micronutrientes também seguiram o método apresentado por esses pesquisadores.

Quadro 1. Características químicas do superfosfato si mples (SS), do fosfato parcialmente aci dulado (FAPS) e do termofosfato magnesiano (TE RM)

\begin{tabular}{|c|c|c|c|c|c|c|}
\hline \multirow{2}{*}{ Adubo } & \multicolumn{3}{|c|}{ Nutrientes totais } & \multicolumn{3}{|c|}{$\mathrm{P}_{2} \mathrm{O}_{5}$ solúvel } \\
\hline & $\mathbf{P}_{2} \mathbf{O}_{5}$ & $\mathbf{S}$ & Mg & $\begin{array}{l}\text { Cit. neutro de } \\
\text { amônio + água }\end{array}$ & $\begin{array}{l}\text { Ác. cítrico } 20 \text { g L-1 } \\
\text { (relação 1:100) }\end{array}$ & $\begin{array}{c}\text { Água } \\
\text { (relação 1:250) }\end{array}$ \\
\hline & & & & $-\mathrm{g} \mathrm{kg}^{-1}$ & & \\
\hline SS & 190 & 111 & - & 190 & 160 & 160 \\
\hline FAPS & 260 & 80 & - & 110 & 110 & 70 \\
\hline TERM & 170 & - & 70 & - & 140 & - \\
\hline
\end{tabular}


Para comparar os três fertilizantes quanto à produção de matéria seca, foi calculado o índice de eficiência agronômica (IEA), de acordo com a fórmula seguinte, usando-se como adubo-referência o superfosfato simples:

$$
\text { IEA }=\frac{P_{r}-P_{o}}{P_{s}-P_{o}} \times 100,
$$

onde: $P_{r}$ : produção de matéria seca obtida com o adubo em teste, na dose $n ; P_{0}$ : produção de matéria seca obtida com o superfosfato simples, na dose $n ; \mathrm{P}_{\mathrm{s}}$ : produção do tratamento sem adubo.

Para comparar os três fertilizantes quanto às quantidades relativas de $\mathrm{P}$ recuperado pela resina, calculou-se o índice de eficiência relativa (IER), de acordo com a fórmula seguinte, usando-se como aduboreferência o superfosfato simples:

$$
\text { IER }=\frac{\mathrm{P} \text { - resina fonte } \mathrm{X}-\mathrm{P} \text { - resina testemunha }}{\mathrm{P}-\text { resina } \mathrm{SS}-\mathrm{P} \text { - resina testemunha }} \times 100,
$$

onde: $\mathrm{P}$-resina fonte $\mathrm{X}: \mathrm{P}$ recuperado pela resina com o adubo em teste, na dose $n$; P-resina testemunha: $P$ recuperado pela resina no tratamento testemunha; $\mathrm{P}$ resina SS: $\mathrm{P}$ recuperado pela resina com o adubo superfosfato simples, na dose $n$.

\section{Incubação de amostras de solo com níveis crescentes de $P$}

Paralelamente ao experimento em casa de vegetação, subamostras de todos os tratamentos, com $2 \mathrm{~kg}$ de solo, foram incubadas em sacos plásticos por $0,10,20,40,60$ e 80 dias, com o teor de água próximo à capacidade de campo. Ao término de cada período de incubação, os sol os eram amostrados e os teores de C-orgânico, P-resina, pH em $\mathrm{CaCl}_{2}$ 0,01 mol L-1 (relação 1:2,5), K, Ca e Mg trocáveis eAl trocável $+\mathrm{H}$ titulável determinados segundo método proposto por Raij et al. (1987).

\section{Análise estatística}

Para a determinação dos níveis críticos de fósforo no solo para a máxima produção de matéria seca, aval iaram-se vários model os de regressão, tendo, como variável dependente, a produção de matéria seca da parte aérea das mudas aos 90 dias pós-emergência, em grama por vaso, e, como variável independente, o teor de P-resina no solo 40 dias após a aplicação dos adubos, em $\mathrm{mg} \mathrm{dm}^{-3}$. Com base no coeficiente de determinação, verificou-se que o mel hor model o foi o quadrático. A comparação entre médias foi feita pelo teste deTukey.

\section{RESULTADOS E DISCUSSÃO}

\section{E feito das fontes de $P$ sobre a composição química dos solos}

As fontes de $P$ afetaram a composição química dos solos de forma bastante distinta, havendo pronunciados efeitos do tempo de incubação sobre a disponibilidade deP e, de modo geral, pequenos efeitos sobre os teores dos demais nutrientes anal isados, que se mantiveram praticamente estáveis desde os períodos iniciais de incubação (Quadro 2).

As porcentagens de fósforo recuperado pelo extrator resina, relativamente às quantidades de $P$ aplicadas, foram, em média, cerca de $10 \%$ menores no LE comparativamente ao AQ (Quadro 3), o que certamente está relacionado com a maior capacidade de fixação de P do LE (Gonçalves et al., 1989, Silva \& Braga, 1993). Cerca de 28 a $60 \%$ das doses de $P$ aplicadas foram recuperadas pela resina na AQ e, 27 a 49\%, no LE. Ao longo do tempo de incubação, para as diferentes fontes e doses, houve oscilações nas quantidades deP extraídas (Quadros 2 e3), atribuídas à capacidade de solubilização das fontes, às reações de adsorção e precipitação de $\mathrm{P}$ e à atividade microbiológica do sol o (mineral ização e imobilização). Contudo, observa-se uma tendência de menor fixação de $\mathrm{P}$ quando a fonte é o superfosfato simples, particularmente no sol o AQ. Neste caso, a fixação de $P$ foi mais de $10 \%$ inferior às demais fontes, que apresentaram entre si níveis de fixação de $\mathrm{P}$ no solo de mesma grandeza.

Quando se compara o índice de eficiência relativa (IER) entreoTERM eoFAPS comoSS, nos diferentes tempos de incubação, verifica-se quea disponibilidade de P cai drasticamente com o tempo de incubação no solo AQ para TERM e FAPS (Quadro 4). As quedas do IER com o tempo de incubação são muito maiores para oFAPS, provavel mente em função da sua menor capacidade de solubilização (relativa à porção de fosfato natural não tratado com ácido sulfúrico) comparativamenteaoTE RM. A situação é inversa no LE, em que o IER do FAPS se el evou com o tempo de incubação, chegando a disponibilidade de $\mathrm{P}$ a ser ligeiramente superior à das amostras que receberam aplicações de SS. I sso reflete a maior solubilização dessa fonte em solos mais argilosos, com maior superfície de adsorção e nível de acidez, por conseguinte, maior remoção da solução do solo dos constituintes do adubo liberados durante sua solubilização, o que intensifica as taxas de dissolução das partículas do adubo.

O TERM foi a única fonte de $\mathrm{P}$ que causou expressiva el evação do pH do solo, redução das concentrações deH +Al eaumento das concentrações de $\mathrm{Mg}$, em ambos os solos (Quadro 2). Esses efeitos foram diretamente proporcionais às doses aplicadas. Resultados semel hantes foram encontrados por Guerrini et al. (1994) e Büll et al. (1995). O SS e o FAPS não alteraram o pH do sol o nem a concentração de $\mathrm{H}+\mathrm{Al}$. Todas as fontes tiveram efeitos similares em relação aos teores de $\mathrm{Ca}$, que teve suas concentracões el evadas de níveis médios equivalentes a 5,0 e $3,0 \mathrm{mmol}_{\mathrm{C}} \mathrm{dm}^{-3}$ para 20,0 e $30,0 \mathrm{mmol}_{\mathrm{C}} \mathrm{dm}^{-3}$, respectivamente, nos solos $A Q$ e LE. A saturação de bases ( $\mathrm{V} \%$ ) el evou-se de níveis médios equival entes a 20 e $10 \%$ para $40 \%$, respectivamente, para ambos os solos, com a aplicação de SS e FAPS (Quadro 2). Todavia, quando oTERM foi aplicado, oV\% subiu para níveis superiores a 70\%, nos dois solos. Esses resultados evidenciam o grande efeito do TERM como corretivo da acidez do solo e fonte de Ca e Mg. É 
Quadro 2. E feito do tempo de incubação do superfosfato simples (SS), fosfato parcialmente acidulado (F APS) e termofosfato magnesiano (TERM) sobre algumas características químicas das amostras de areia quartzosa (AQ) e latossolo vermelho-escuro (LE ). Na AQ, for am aplicados $150 \mathrm{mg} \mathrm{dm}^{-3} \mathrm{de}^{\mathrm{P}}$ no solo e, no LE , $250 \mathrm{mg} \mathrm{kg}^{-1}$ de $P$ no solo

\begin{tabular}{|c|c|c|c|c|c|c|c|c|c|}
\hline \multirow{2}{*}{ Solo } & \multirow{2}{*}{ Fonte de $\mathbf{P}$} & \multicolumn{8}{|c|}{ Tempo de incubação (dias) } \\
\hline & & $\mathbf{0}$ & 10 & 40 & 80 & $\mathbf{0}$ & 10 & 40 & 80 \\
\hline & & \multicolumn{4}{|c|}{ pH em $\mathrm{CaCl}_{2} 0,01 \mathrm{~mol} \mathrm{~L}^{-1}$} & \multicolumn{4}{|c|}{ P-resina ( $\left.\mathrm{mg} \mathrm{dm}^{-3}\right)$} \\
\hline AQ & $\begin{array}{l}\text { TERM } \\
\text { FAPS } \\
\text { S S } \\
\text { Testemunha }\end{array}$ & $\begin{array}{l}5,4 \\
3,6 \\
3,6 \\
3,6\end{array}$ & $\begin{array}{l}5,6 \\
3,9 \\
3,9 \\
3,9\end{array}$ & $\begin{array}{l}5,8 \\
3,8 \\
3,8 \\
3,8\end{array}$ & $\begin{array}{l}5,5 \\
3,8 \\
3,8 \\
3,9\end{array}$ & $\begin{array}{l}93 \\
85 \\
94 \\
13\end{array}$ & $\begin{array}{l}60 \\
90 \\
77 \\
18\end{array}$ & $\begin{array}{r}68 \\
56 \\
103 \\
13\end{array}$ & $\begin{array}{r}75 \\
62 \\
101 \\
12\end{array}$ \\
\hline \multirow[t]{2}{*}{ LE } & $\begin{array}{l}\text { TERM } \\
\text { FAPS } \\
\text { S S } \\
\text { Testemunha }\end{array}$ & $\begin{array}{l}5,4 \\
3,7 \\
3,7 \\
3,8\end{array}$ & $\begin{array}{l}5,8 \\
4,1 \\
4,0 \\
4,0\end{array}$ & $\begin{array}{l}5,7 \\
4,0 \\
4,0 \\
4,0\end{array}$ & $\begin{array}{l}5,0 \\
4,0 \\
4,0 \\
3,9\end{array}$ & $\begin{array}{r}96 \\
100 \\
126 \\
6\end{array}$ & $\begin{array}{r}83 \\
79 \\
98 \\
6\end{array}$ & $\begin{array}{r}85 \\
115 \\
111 \\
7\end{array}$ & $\begin{array}{r}72 \\
91 \\
91 \\
6\end{array}$ \\
\hline & & \multicolumn{4}{|c|}{$K\left(\mathrm{mmol}_{\mathrm{c}} \mathrm{dm}^{-3}\right)$} & \multicolumn{4}{|c|}{$\mathrm{Ca}\left(\mathrm{mmol}_{\mathrm{c}} \mathrm{dm}^{-3}\right)$} \\
\hline AQ & $\begin{array}{l}\text { TERM } \\
\text { FAPS } \\
\text { S S } \\
\text { Testemunha }\end{array}$ & $\begin{array}{l}3,7 \\
3,6 \\
3,7 \\
4,0\end{array}$ & $\begin{array}{l}5,0 \\
5,3 \\
4,8 \\
5,2\end{array}$ & $\begin{array}{l}4,8 \\
4,2 \\
3,8 \\
3,8\end{array}$ & $\begin{array}{l}4,0 \\
4,0 \\
3,4 \\
3,0\end{array}$ & $\begin{array}{r}18,4 \\
22,8 \\
19,2 \\
5,5\end{array}$ & $\begin{array}{r}17,4 \\
20,7 \\
16,9 \\
4,2\end{array}$ & $\begin{array}{r}21,2 \\
22,6 \\
22,0 \\
8,4\end{array}$ & $\begin{array}{r}20,0 \\
17,0 \\
21,0 \\
2,0\end{array}$ \\
\hline LE & $\begin{array}{l}\text { TERM } \\
\text { FAPS } \\
\text { S S } \\
\text { Testemunha }\end{array}$ & $\begin{array}{l}3,8 \\
4,9 \\
4,3 \\
3,7\end{array}$ & $\begin{array}{l}4,7 \\
4,7 \\
5,2 \\
4,7\end{array}$ & $\begin{array}{l}4,1 \\
3,7 \\
4,6 \\
4,8\end{array}$ & $\begin{array}{l}3,2 \\
4,4 \\
3,2 \\
4,4\end{array}$ & $\begin{array}{r}31,0 \\
31,2 \\
33,1 \\
2,3\end{array}$ & $\begin{array}{r}31,8 \\
27,1 \\
28,2 \\
2,3\end{array}$ & $\begin{array}{r}32,0 \\
35,4 \\
34,3 \\
2,8\end{array}$ & $\begin{array}{r}32,0 \\
27,0 \\
31,0 \\
3,0\end{array}$ \\
\hline & & \multicolumn{4}{|c|}{$\mathrm{Mg}\left(\mathrm{mmol}_{\mathrm{c}} \mathrm{dm}^{-3}\right)$} & \multicolumn{4}{|c|}{$\mathrm{H}+\mathrm{Al}\left(\mathrm{mmol}_{\mathrm{c}} \mathrm{dm}^{-3}\right)$} \\
\hline AQ & $\begin{array}{l}\text { TERM } \\
\text { FAPS } \\
\text { S S } \\
\text { Testemunha }\end{array}$ & $\begin{array}{r}18,2 \\
0,9 \\
1,5 \\
0,9\end{array}$ & $\begin{array}{r}12,9 \\
1,1 \\
1,4 \\
0,8\end{array}$ & $\begin{array}{r}17,2 \\
0,9 \\
6,1 \\
1,1\end{array}$ & $\begin{array}{r}17,4 \\
1,2 \\
1,7 \\
0,7\end{array}$ & $\begin{array}{l}20,0 \\
42,0 \\
42,0 \\
38,0\end{array}$ & $\begin{array}{l}16,0 \\
34,0 \\
34,0 \\
34,0\end{array}$ & $\begin{array}{l}13,0 \\
31,0 \\
31,0 \\
31,0\end{array}$ & $\begin{array}{l}15,0 \\
34,0 \\
38,0 \\
31,0\end{array}$ \\
\hline LE & $\begin{array}{l}\text { TERM } \\
\text { FAPS } \\
\text { S S } \\
\text { Testemunha }\end{array}$ & $\begin{array}{r}24,1 \\
1,5 \\
1,9 \\
0,9\end{array}$ & $\begin{array}{r}23,7 \\
1,2 \\
2,3 \\
0,6\end{array}$ & $\begin{array}{r}26,3 \\
1,6 \\
1,9 \\
0,7\end{array}$ & $\begin{array}{r}25,6 \\
1,2 \\
2,1 \\
0,6\end{array}$ & $\begin{array}{l}28,0 \\
64,0 \\
64,0 \\
64,0\end{array}$ & $\begin{array}{l}22,0 \\
52,0 \\
58,0 \\
58,0\end{array}$ & $\begin{array}{l}18,0 \\
52,0 \\
52,0 \\
52,0\end{array}$ & $\begin{array}{l}28,0 \\
52,0 \\
52,0 \\
52,0\end{array}$ \\
\hline
\end{tabular}

Quadro 3. Percentagem média de fósforo recuperado(1) pela resina, após diferentes períodos de incubação das amostras de solo. Na AQ, foram aplicados $150 \mathrm{mg} \mathrm{kg}^{-1}$ de P no solo e, no LE , $250 \mathrm{mg} \mathrm{kg}^{-1}$ de P no solo

\begin{tabular}{|c|c|c|c|c|c|c|c|c|}
\hline \multirow{2}{*}{$\begin{array}{l}\text { Fonte } \\
\text { deP }\end{array}$} & \multicolumn{6}{|c|}{ Período de incubação (dias) } & \multirow{2}{*}{ Médias } & \multirow{2}{*}{$\mathbf{S}^{(3)}$} \\
\hline & $\mathbf{0}$ & 10 & 20 & 40 & 60 & 80 & & \\
\hline & & & & reia & uartz & & & \\
\hline \multirow[t]{2}{*}{$\begin{array}{l}\text { TERM } \\
\text { FAPS } \\
\text { SS }\end{array}$} & $\begin{array}{l}53 \\
47 \\
53\end{array}$ & $\begin{array}{l}28 \\
48 \\
39\end{array}$ & $\begin{array}{l}38 \\
31 \\
31\end{array}$ & $\begin{array}{l}36 \\
29 \\
60\end{array}$ & $\begin{array}{l}32 \\
44 \\
57\end{array}$ & $\begin{array}{l}42 \\
33 \\
60\end{array}$ & $\begin{array}{l}40 \\
39 \\
53\end{array}$ & $\begin{array}{l}10 \\
10 \\
10\end{array}$ \\
\hline & \multicolumn{8}{|c|}{ Latossolo vermelho-escuro } \\
\hline $\begin{array}{l}\text { TERM } \\
\text { FAPS } \\
\text { SS }\end{array}$ & $\begin{array}{l}36 \\
38 \\
49\end{array}$ & $\begin{array}{l}31 \\
30 \\
37\end{array}$ & $\begin{array}{l}42 \\
26 \\
24\end{array}$ & $\begin{array}{l}31 \\
43 \\
42\end{array}$ & $\begin{array}{l}31 \\
29 \\
31\end{array}$ & $\begin{array}{l}27 \\
34 \\
34\end{array}$ & $\begin{array}{l}32 \\
36 \\
41\end{array}$ & $\begin{array}{l}4 \\
6 \\
7\end{array}$ \\
\hline \multicolumn{9}{|c|}{$\%$ de $\mathrm{P}$ recuperado $=\frac{(\mathrm{P}-\text { resina }-\mathrm{P}-\text { resina testemunha })}{\mathrm{P}-\text { aplicado }} \times 100$} \\
\hline
\end{tabular}

importante destacar que a maioria das espécies de eucal ipto usadas no Brasil são plenamenteadaptadas a solos distróficos, sendo pouco sensíveis à acidez do solo e a altas saturações de Al (N ovais et al., 1982, Gonçalves et al., 1997). O eucalipto responde à calagem somente quando os níveis de Ca e Mg no sol o são limitantes para o crescimento das plantas (Gonçalves et al., 1996). Por conseguinte, suprimidas as deficiências de $P$, esperam-se efeitos pronunciados das fontes fosfatadas, que el evam os teores de $\mathrm{Ca}$ e Mg quando os níveis desses nutrientes no solo são baixos, seja pelo suprimento adicional de $\mathrm{Ca}$ e $\mathrm{Mg}$, seja pelos efeitos benéficos que tais nutrientes poderiam ter sobre a absorção de outros nutrientes essenciais para as plantas, sob níveis de $\mathrm{pH}$ menos ácidos (quando a fonte for o termofosfato).

Eficiência relativa das fontes de $\mathbf{P}$ avaliada pela produção de biomassa

A resposta das plantas à aplicação de $P$ foi generalizada, sendo significativa mesmo nas suas 
Quadro 4. Índice de eficiência relativa (IE R) entre o termofosfato magnesiano (TERM) e o fosfato parcialmente acidulado (FAPS) relativamente ao superfosfato simples (SS), para diferentes tempos de incubação

\begin{tabular}{|c|c|c|c|c|c|}
\hline \multirow{2}{*}{$\begin{array}{c}\text { Fonte } \\
\text { de P }\end{array}$} & \multirow{2}{*}{$\begin{array}{l}\text { Dose } \\
\text { de P }\end{array}$} & \multicolumn{4}{|c|}{ Tempo de incubação (dias) } \\
\hline & & $\mathbf{0}$ & 10 & 40 & 80 \\
\hline & \multirow[t]{2}{*}{$\mathrm{mg} \mathrm{dm} 3$} & & 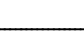 & . & - \\
\hline & & \multicolumn{4}{|c|}{ Areia quartzosa } \\
\hline TERM & 150 & 99 & 71 & 61 & 71 \\
\hline \multirow[t]{2}{*}{ FAPS } & 150 & 89 & 122 & 48 & 56 \\
\hline & & \multicolumn{4}{|c|}{ Latossolo vermelho-escuro } \\
\hline TERM & 250 & 75 & 84 & 75 & 76 \\
\hline FAPS & 250 & 78 & 79 & 104 & 700 \\
\hline
\end{tabular}

menores doses. OTERM foi o adubo que proporcionou o mai or crescimento das mudas de E. grandis no solo AQ. Por outro lado, foi a pior fonte no LE (Figura 1). Neste, a melhor fonte foi o SS. O FAPS teve comportamento semel hante ao SS no AQ e intermediário entre as demais fontes no LE. Utilizando oSS como fonte dereferência para o cálculo doíndice de eficiência agronômica (IEA), verificou-se que oTERM foi superior $64 \%$ ao SS e $57 \%$ ao FAPS no solo AQ. No solo LE, oSS foi superior $55 \%$ aoTERM e $34 \%$ ao FAPS. É importante destacar que, independentemente da resposta aos adubos aplicados, os níveis de produção de matéria seca do LE foram, em média, muito superiores ao AQ, apesar de os teores de $P, K$ e Ca não serem muito diferentes entre esses solos, após a aplicação das fontes de P. Por outro lado, os maiores ganhos relativos de produção de matéria seca, quando se compara a mel hor fonte de P com as demais, foram conseguidos no solo $\mathrm{AQ}$.

De modo geral, as doses de $\mathrm{P}$ aplicadas foram altamente correlacionadas com as concentrações de nutrientes na parte aérea das mudas de eucali pto (Quadro 5). Considerando que as quantidades de biomassa produzidas nos diferentes tratamentos foram altamente relacionadas com as quantidades aplicadas e recuperadas de $\mathrm{P}$ (Figura 1), conclui-se que as diferenças de crescimento observadas entre os tratamentos estão altamente relacionadas com as quantidades de $\mathrm{P}, \mathrm{K}, \mathrm{Ca}$, M ge Zn absorvidas. OTERM proporcionou as maiores absor ções desses nutrientes no solo AQ e as menores absorções de P, Ca e Zn no LE (Quadro 6). Esses efeitos sobre o crescimento não devem ter relação direta com a disponibilidade de $P$ nosolo, por si só, pois os teores deP no solo resultantes da aplicação das duas doses mais el evadas de adubo atingiram níveis adequados para o crescimento das mudas em ambos os solos, para todas as fontes de $P$ usadas. Nessa linha de raciocínio, a maior resposta ao TERM no AQ está relacionada com a melhoria global do nível de fertilidade do solo, comojá discutido.

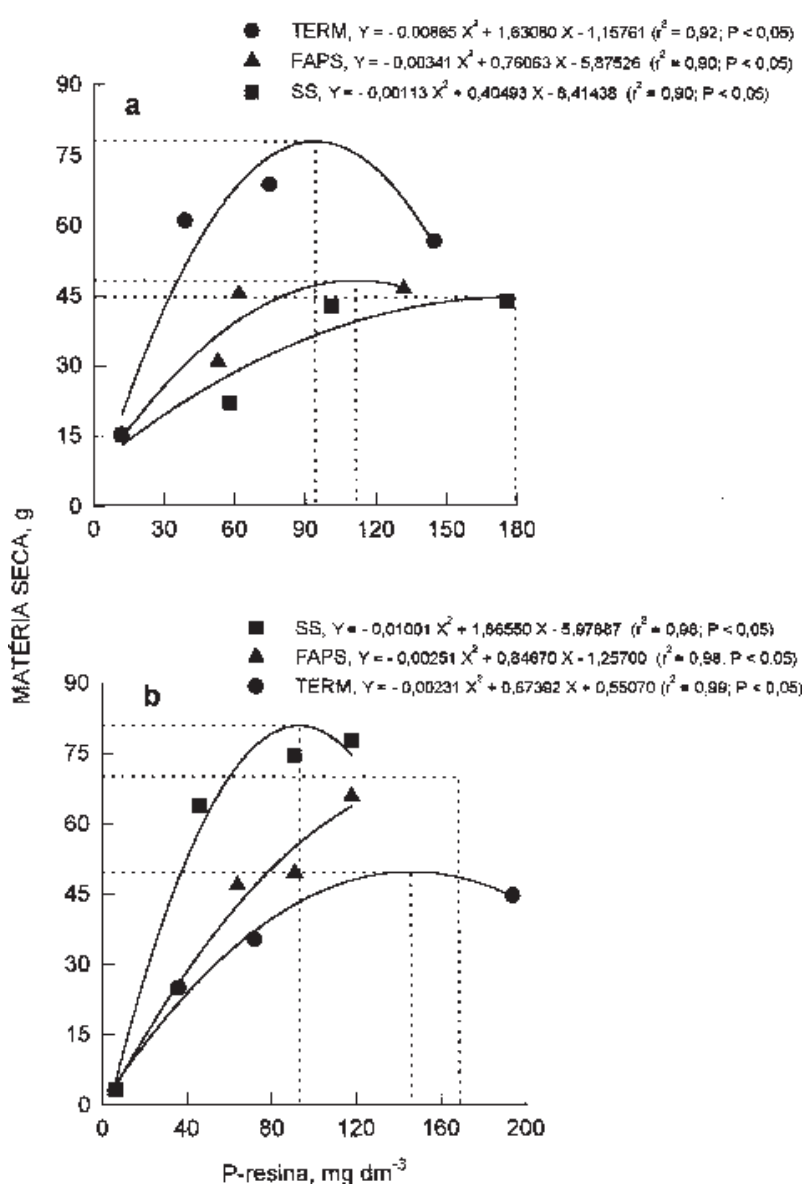

Figura 1. Crescimento das mudas de eucalipto aos 90 dias pós-emergência em função das concentrações de $\mathbf{P}$ recuperadas pela resina. As linhas descontínuas destacam os níveis críticos de $\mathbf{P}$, para as diferentes fontes de $\mathbf{P}$ e solos (a) areia quartzosa e (b) latossolo vermelho-escuro, responsáveis pelas produções máximas de matéria seca da parte aérea. 0 AQ recebeu $0,75,150$ e $300 \mathrm{mg} \mathrm{kg}^{-1}$ de $P$ e, o LE, 0, 125, 250 e $500 \mathrm{mg} \mathrm{kg}^{-1}$ de $P$ no solo. E ssas doses foram estimadas com base no teor de $\mathbf{P}$ solúvel dos adubos.

Quadro 5. Correlação entre as doses de P aplicadas e as concentrações de nutrientes na parte aérea de mudas de E ucal yptus grandis aos 90 dias pósemergência, para os diferentes tipos de solo e fontes de $\mathbf{P}(1)$

\begin{tabular}{|c|c|c|c|c|c|c|}
\hline Solo & $\begin{array}{c}\text { Fonte } \\
\text { de } P\end{array}$ & $\mathbf{P}$ & $\mathbf{K}$ & $\mathrm{Ca}$ & Mg & Zn \\
\hline $\mathrm{AQ}$ & $\begin{array}{l}\text { TERM } \\
\text { FAPS } \\
\text { SS }\end{array}$ & $\begin{array}{l}0,82^{* *} \\
0,90^{* *} \\
0,73^{* *}\end{array}$ & $\begin{array}{l}-0,32^{\mathrm{ns}} \\
-0,12^{\mathrm{ns}} \\
-0,04^{\mathrm{ns}}\end{array}$ & $\begin{array}{l}0,38^{\text {ns }} \\
0,57^{*} \\
0,27^{\text {ns }}\end{array}$ & $\begin{array}{c}0,81^{* *} \\
-0,56^{*} \\
-0,25^{\text {ns }}\end{array}$ & $\begin{array}{c}-0,51^{\text {ns }} \\
0,54^{*} \\
0,48^{\text {ns }}\end{array}$ \\
\hline LE & $\begin{array}{l}\text { TERM } \\
\text { FAPS } \\
\text { SS }\end{array}$ & $\begin{array}{l}0,77^{* *} \\
0,59^{*} \\
0,91^{* *}\end{array}$ & $\begin{array}{l}-0,72^{* *} \\
-0,73^{* *} \\
-0,78^{* *}\end{array}$ & $\begin{array}{l}0,40^{\text {ns }} \\
0,67^{* *} \\
0,79^{* *}\end{array}$ & $\begin{array}{c}0,87^{* *} \\
-0,52^{\text {ns }} \\
-0,61^{*}\end{array}$ & $\begin{array}{l}-0,89^{* *} \\
-0,77^{* *} \\
-0,43^{n s}\end{array}$ \\
\hline
\end{tabular}

(1) TERM : termofosfato magnesiano; FAPS: fosfato parcialmente acidulado; SS: superfosfato simples. ${ }^{\text {ns }}=$ não significativo; ${ }^{*}=$ significativo ao nível de $5 \%$ de probabilidade; $e^{* *}=$ significativo ao nível de $1 \%$ de probabilidade pelo teste de Tukey. 
Quadro 6. Quantidades totais de P, K, Ca, Mg e Zn contidas na matéria seca da parte aérea das mudas de Eucalyptus grandis aos $\mathbf{9 0}$ dias de idade, em função do tipo de solo e fonte, quando aplicados 150 e $250 \mathrm{mg} \mathrm{kg}^{-1}$ de P no solo. Valores seguidos pela mesma letra não diferem estatisticamente pelo teste de Tukey $(P=0,05)$

\begin{tabular}{lccccc}
\hline & P & K & Ca & Mg & Zn \\
\cline { 2 - 6 } & \multicolumn{6}{c}{ mg/vaso } \\
\cline { 2 - 6 } TERM & $96 \mathrm{a}$ & $344 \mathrm{a}$ & $440 \mathrm{a}$ & $234 \mathrm{a}$ & $4,8 \mathrm{a}$ \\
FAPS & $59 \mathrm{~b}$ & $226 \mathrm{~b}$ & $285 \mathrm{~b}$ & $50 \mathrm{~b}$ & $2,3 \mathrm{~b}$ \\
S S & $81 \mathrm{c}$ & $124 \mathrm{c}$ & $559 \mathrm{c}$ & $85 \mathrm{c}$ & $4,1 \mathrm{c}$ \\
& \multicolumn{7}{c}{ Latossolo vermelho-escuro } \\
TERM & $56 \mathrm{~b}$ & $201 \mathrm{~b}$ & $321 \mathrm{~b}$ & $120 \mathrm{~d}$ & $1,7 \mathrm{a}$ \\
FAPS & $59 \mathrm{~b}$ & $213 \mathrm{~b}$ & $544 \mathrm{c}$ & $74 \mathrm{c}$ & $2,6 \mathrm{~b}$ \\
S S & $82 \mathrm{c}$ & $320 \mathrm{a}$ & $677 \mathrm{~d}$ & $97 \mathrm{c}$ & $3,9 \mathrm{c}$ \\
\hline
\end{tabular}

(1) TERM: termofosfato magnesiano; FAPS: fosfato parcialmente acidulado; SS: superfosfato simples.

Os maiores valores de nível crítico encontrados no LE (Figura 1), paraTERM eFAPS, estão relacionados com o maior potencial produtivo e com a maior superfície de adsorção de $\mathrm{P}$, relativamente ao AQ. Observa-se na figura 1 que, para as melhores fontes de $P$ nos dois solos em estudo (TERM no AQ e SS no LE), os ganhos máximos de produção, em relação ao tratamento sem adubo, foram maiores no LE, apesar de o nível crítico deP ter sido idêntico em ambos para essas fontes.

Por fim, sabendo-se que, para os três adubos avaliados, aplicaram-se doses semelhantes de $P$ solúvel eque os níveis deP disponível encontrados no solo para as maiores doses aplicadas são adequados para o crescimento de mudas de eucalipto (Novais et al., 1990), conclui-se que as distintas respostas de crescimento para os adubos avaliados em ambos os solos, sobretudo para oTERM e oSS, são devidas, em grande parte, aos efeitos secundários das fontes de $P$ sobre a fertilidade dos solos. Além disso, sabendo-se que significativa fração das formas de $P$ encontradas no FAPS e TERM são de lenta a moderada solubilização, os efeitos observados nesta pesquisa poderiam ser bastante diferentes se o período de experimentação fosse mai or, principalmente se as condições de trabal ho fossem as de campo. Contudo, considerando que as fontes solúveis de $\mathrm{P}$ se aplicam de forma localizada no campo (na cova ou sulco de plantio) e que um dos períodos mais críticos para o estabel ecimento de plantações de eucalipto são os três a seis meses iniciais de crescimento, deduz-se que as informações obtidas nesta pesquisa (sob condições de casa de vegetação) devem ter grande relação com o crescimento das mudas em resposta aos adubos avaliados sob condições de campo.

\section{CONCLUSÕES}

1. As porcentagens de $P$ recuperadas pel oextrator resina relativamente às quantidades de $\mathrm{P}$ aplicadas foram, em média, cerca de 10\% menores no LE, comparativamente ao AQ.

2. O termofosfato foi o adubo que proporcionou o maior crescimento das mudas de $\mathrm{E}$. grandis no solo AQ. Por outro lado, foi a pior fonte no LE. Neste, a mel hor fonte foi o superfosfato simples. Por meio do cálculo de índices de eficiência relativa entre os adubos, verificou-se que o termofosfato foi superior $64 \%$ ao superfosfato simples e $57 \%$ ao FAPS no solo AQ. No LE, o superfosfato simples foi superior $55 \%$ ao termofosfato e $34 \%$ ao FAPS.

3. O termofosfato magnesiano foi a fonte de $P$ que causou os maiores acréscimos do nível de fertilidade dos solos, com expressiva el evação do pH do solo, redução das concentrações de Al e aumento das concentrações de $\mathrm{Ca}$ e $\mathrm{Mg}$. Esses resultados evidenciam o grande efeito do termofosfato magnesiano como corretivo da acidez do solo e fonte de Ca e Mg para eucalipto, sobretudo em sol os mais arenosos e de baixa fertilidade.

4. Os ganhos de produção das mudas de eucal ipto foram altamente correlacionados com a absorção de $\mathrm{P}, \mathrm{K}, \mathrm{Ca}, \mathrm{Mg}$ e $\mathrm{Zn}$. O termofosfato proporcionou as maiores absorções desses nutrientes no solo $\mathrm{AQ}$ e as menores absorções de P, Ca e Zn no LE.

5. Os maiores níveis críticos de $P$ no LE para as fontes TERM e FAPS foram atribuídos a seu maior potencial produtivo, a maior acidez e superfície de adsorção de $\mathrm{P}$, relativamente ao AQ.

6. As distintas respostas de crescimento para os adubos avaliados, em ambos os solos, sobretudo para oTERM e o SS, foram atribuídas, em grande parte, aos efeitos secundários das fontes de $\mathrm{P}$ sobre a fertilidade dos solos.

\section{AGRADE CIMENTOS}

À FAPESP, pela bolsa de iniciação científica concedida ao primeiroautor desta pesquisa, quemuito contribuiu para seu aprimoramento profissional .

\section{LITE RATURA CITADA}

BALLARD, R. Fertilization of plantations. In: BOWEN, G.D. \& NAMBIAR, E.K.S. Nutrition of plantation forests. London, Academic Press, 1984. p.327-60.

BARROS, N.F.; NOVAIS, R.F. \& NEVES, J.C.L. Fertilização e correção do solo para o plantio de eucalipto. In: BARROS, N.F. \& NOVAIS, R.F., eds., Relação solo-eucalipto. Viçosa, Editora Fol ha de Viçosa, 1990. p.127-186.

BÜLL, L.T.; LACERDA, S. \& NAKAGAWA, J. Alterações em propriedades químicas no solo e eficiência agronômica de termofosfatos. In: CONGRESSO BRASILEIRO DE CIÊNCIA DO SOLO, 25., Viçosa, 1995. Resumos Expandidos. Viçosa, SBCS, 1995. p. 2217-2219. 
DANTAS, S.V. Avaliação da eficiência de fosfatos em plantios de Eucalyptus grandis. Viçosa, Universidade Federal deViçosa, 1988. 141p. (Tese de Doutorado)

GOEDERT, W.J . \& SOUZA, D.M.G. Uso eficiente de fertilizantes fosfatados. In: SIMPÓSIO SOBRE FERTILIZANTES NA AGRICULTURA BRASILEIRA, Brasília, 1984. Anais. Brasília, EMBRAPA, 1984. p.255-289.

GONÇALVES, J.L.M. Recomendações de adubação para Eucalyptus, Pinus e espécies típicas da Mata Atlântica. Documentos Florestais, Piracicaba, 15:1-23, 1995.

GONÇALVES, J.L.M.; BARROS, N.F.; NEVES, J.C.L. \& NOVAIS, R.F. Níveis críticos de fósforo no solo e na parte aérea de eucalipto na presença e ausência de calagem. Rev. Árv., Viçosa, 10:91-104, 1996.

GONÇALVES, J.L.M.; BARROS, N.F.; NAMBIAR, E.K.S. \& NOVAIS, R.F. Soil and stand management for intensive shortrotation plantations. In: NAMBIAR, S. \& BROWN, A., eds. Management of soil, water and nutrients in tropical plantation forests. Canberra, CSIRO/ACIAR/CIFOR, 1997. cap.11. (no prelo)

GONÇALVES, J.L.M.; NOVAIS, R.F.; BARROS, N.F.; NEVES, J.C.L. \& RIBEIRO, A.C. Cinética de transformação de fósforo lábil em nãolábil, em solos de Cerrado. R. bras. Ci. Solo, Campinas, 13:13-224, 1989.

GUERRINI, I.A.; VILLAS BÔAS, R.L.; KORMAN, V. \& YASUDA, $M$. E feito de fontes de fósforo sobre o desenvolvimento inicial de Eucalyptus grandis. In: REUNIÃO BRASILEIRA DE FERTILIDADE DO SOLO E NUTRIÇÃO DE PLANTAS, 21. Petrolina, 1994. Anais. Petrolina, SBCS, 1994. p.10-11.
HERBERT, M.A. The response of Eucalyptus grandis to fertilising with nitrogen, phosphorus, potassium and dolomitic lime on a Mispah soil series. South Afr. For. J., Pretoria, 124:4-12, 1983.

HERBERT, M.A. \& SCHÖNAU, A.P.G. Fertilising commercial forest species in Southern Africa: research progress and problems. Pt.1. South Afr. For. J., Pretoria, 151:58-70, 1989.

LEAL, P.G.L.; BARROS, N.F.; NOVAIS, R.F.; NEVES, J.C.L. \& TEXEIRA, J.L. Biomassa e conteúdo de nutrientes em Eucalyptus grandis influenciados pela aplicação de fosfatos naturais em solos de cerrado. Rev. Árv., Viçosa, 12:165-182, 1988.

NOVAIS, R.F.; BARROS, N.F. \& NEVES, J.C.L. Nutrição mineral do eucalipto. In: BARROS, N.F \& NOVAIS, R.F., eds. Relação solo-eucalipto. Viçosa, Editora Fol ha de Viçosa, 1990. 330p.

NOVAIS, R.F.; BARROS, N.F.; NEVES, J.C.L. \& COUTO, C. Níveis críticos de fósforo para o eucalipto. Rev. Árv., Viçosa, 6: 2937, 1982.

RAIJ, B. VAN; QUAGGIO, J.A.; CANTARELLA, H.; FERREIRA, M.E.; LOPES, A.S. \& BATAGLIA, O.C. Análise química do solo para fins de fertilidade. Campinas, Fundação Cargill, 1987. 170p.

REZENDE, G.V.; BARROS, N.F.; MORAES, T.S.A.; MENDES, C.J . $\&$ SUITER FILHO, W. Aplicação de fosfatos naturais em plantios de Eucalyptus grandis W. Hill ex Maiden. Rev. Árv., Viçosa, 6:74-83, 1982.

SARRUGE, J.R. \& HAAG, H.P. Análise química em plantas. Piracicaba, ESALQ, 1974. 56p. 